\title{
Taking stock in the New Year
}

As 2014 begins, we look back at our performance over the previous year.

$\mathbf{E}$ at more healthily. Exercise more. Learn to play a musical instrument. Finally read that (in)famous book and find out what the fuss is all about. Many of us dutifully embrace some of these common New Year's resolutions-even if, come March, we might push that book, spine intact, far back on the bottom shelf.

As editors at Nature Structural \& Molecular Biology, we have but one resolution: to provide the best service for our scientific communities, including readers and authors alike. This is not an elusive, distant goal; it is our concrete mission, to be accomplished with each and every manuscript that we handle. More specifically, we aim to publish papers that are of broad interest, that provide new mechanistic and biological insight and that will stimulate discussion and further research. We want the data in our papers to be sound and robust, clearly presented and useful to readers. Last, but not least, we do our best to process our manuscripts in a fair, consistent and efficient manner

To do all this and to improve our performance, we regularly monitor our workload and manuscript processing times. We are frequently asked about this information by authors and readers, so here we share some 2013 numbers with you.

The NSMB editorial team is composed of full-time editors-all with doctorates and extensive research experience-who assess all manuscripts and make all decisions. To clear up a common misconception: we do not have an editorial board. This ensures our editorial independence-our sole vested interest is to publish the best papers. When hesitant about a new manuscript, we do occasionally consult an outside expert (and potential referee). We use a broad and diverse pool of reviewers: in 2013, nearly 600 scientists acted as reviewers for $N S M B$, and the vast majority (over $80 \%$ ) reviewed a single manuscript.

New manuscripts are assigned to an editor, usually within one working day after submission. The editor assesses the work and discusses it with the team, and a decision is then made on whether to send the manuscript out for peer review. This is a highly stringent step. Of all new submissions to NSMB in 2013, 15\% were sent to referees. (We should emphasize that this is not a predetermined quota, and the specific rate may differ according to field.) This selection may seem overly harsh, but with about 120 new submissions each month, we must make those first, tough editorial calls. But we do not make them lightly. In fact, contrary to popular belief, every manuscript submitted to NSMB is read and evaluated in full. We neither triage manuscripts nor make decisions on the basis of titles, abstracts or who the authors are. And although we are thorough when considering new manuscripts, we also try to be fast. In 2013, our average time for this first decision was 3.5 calendar days after assignment of a submission to an editor.
Still, rejection stings, and we receive a fair number of complaints and appeals from authors at this stage. However, of those submissions that went through peer review in 2013, slightly more than half (56\%) had a positive outcome; a handful are still undergoing review or revision. But beware-this doesn't mean that appealing and trying to make us send your manuscript to referees will result in a high chance of being published in NSMB. In fact, appeals of initial editorial decisions are not highly successful, and only $20 \%$ of the appeals for which we sought input from reviewers fared well and were eventually published. Thus, although first impressions may not always be correct, these numbers suggest that our initial judgment is fairly accurate.

In 2013, authors waited, on average, 36 days after editor assignment for the decision after the first round of review. This time period (which includes the aforementioned 3.5 days for the decision to review the manuscript) can be broken down into a number of additional steps. The first is to obtain the reproducibility checklist (and structure validation reports, if applicable) from the authors, policies that were introduced in the May 2013 editorial. This can take several days, and therefore we encourage authors to send those files together with the original submission. Next, the editor identifies and engages appropriate reviewers; this might take a week or sometimes longer, depending on the responsiveness and responses from prospective reviewers, who are asked to return reports within 2 weeks. Once reviewers' reports are available, the handling editor assesses and discusses them with the editorial team before reaching a decision; this typically takes place within 2 days.

Many authors get anxious or frustrated during the review process, and we take measures to move things along. We avoid reviewers who tend to be tardy or, worse, who have pulled disappearing acts in the past. We make decisions without waiting for a late reviewer whenever feasible (e.g., when the particular expertise of the late reviewer overlaps with that of another reviewer). If a reviewer's report seems exceptionally severe, we might consult one of the other reviewers before making a decision. When inviting a revision, we clearly identify the major issues that need to be addressed and are always available to discuss how to best respond to reviewers' concerns. Once authors submit their revision and response, we involve reviewers again only when necessary (e.g., to assess more technical points or evaluate new data). In cases of looming competition, we expedite the review process, and after a paper is accepted we do our best to publish it as quickly as possible.

We consider our main role as editors to work together with authors in improving their manuscripts, as that is ultimately the whole purpose of the review process. We look forward to working with you in the New Year, and we hope that the information here will give you reassurance when you place your next manuscript in our hands. 